\title{
Manifestación emocional y modelación de una función
}

\author{
matemática
}

\section{Emotional manifestation and modeling of a mathematical function}

\author{
Verónica Díaz* \\ ORCID iD 0000-0001-6428-2711 \\ Hector Belmar** \\ ORCID iD 0000-0002-0754-7098 \\ Álvaro Poblete*** \\ ORCID iD 0000-0003-0907-5095
}

\begin{abstract}
Resumen
Esta investigación vincula aspectos cognitivos y afectivos en la resolución de una situación de aprendizaje, con el propósito de conocer la manifestación emocional que tienen los estudiantes en el proceso de aprehender la modelación del objeto matemático función lineal. Con ello se pretende contribuir en el campo del dominio afectivo, dada la relevancia que en las últimas décadas se está dando a esta dimensión en la Educación Matemática, por constituir un factor clave en la comprensión del rendimiento en la materia. Para ello, se realizó una investigación de carácter descriptiva con metodología cuantitativa con una muestra de 349 estudiantes de la enseñanza secundaria, pertenecientes a seis colegios de una región de Chile. Se utilizó la modelación matemática entendiéndola como un intento de describir una parte del mundo real en términos matemáticos y los resultados indican que el nexo entre las variables rendimiento en Matemáticas y emocionalidad es evidente, no así la relación género y emociones.
\end{abstract}

Palabras clave: Emociones. Modelación matemática. Resolución de problemas. Educación secundaria.

\begin{abstract}
This research links cognitive and affective variables in the resolution of a learning situation, with the aim of knowing the students' emotional manifestation during the process of apprehending the modeling of the mathematical object linear function. This study intends to contribute to the field of affective domain in Mathematics Education, given the relevance this dimension has had in the last decades, because it is a key factor in understanding performance in this area. With this in mind, a descriptive research study was carried out using a quantitative methodology with a sample of 349 secondary school students from six schools in a Chilean region. Mathematical modeling was used as an attempt to describe part of the real world in mathematical terms; results

\footnotetext{
* Doctora en Educación con Especialización en Matemática por la Universidad Humanismo Cristiano (UAHC). Académica e investigadora del Departamento de Ciencias Exactas de la Universidad de Los Lagos (ULA), Osorno, Región de Los Lagos, Chile. Dirección postal: Casilla 933, Depto. Ciencias Exactas, Osorno, Chile, C.P: 5290000. E-mail: mvdiaz@ulagos.cl.

** Mg. en educación Matemática por la Universidad de Los Lagos (ULA). Ingeniero y Académico de la Universidad Tecnológica de Chile, Santiago, Región Metropolitana, Chile. Dirección postal: Puerto Montt, 18.368, ciudad Satélite, Maipú, Región Metropolitana, Chile. E-mail: hector.belmar@hotmail.com.

** Ph.D en Didáctica Matemática por la Université Laval (UL). Académico e investigador del Departamento de Ciencias Exactas de la Universidad de Los Lagos (ULA), Osorno, Región de Los Lagos, Chile. Dirección postal: Casilla 933, Depto. Ciencias Exactas, Osorno, Chile, C.P: 5290000. E-mail: apoblete@ulagos.cl.
} 
indicate that the nexus between performance in Mathematics and emotionality variables is evident, but not the relation between gender and emotions.

Keywords: Emotions. Mathematical Modeling. Problem solving. Secondary Education.

\section{Introducción}

La investigación que se presenta tiene sus raíces en la evolución e importancia que educadores y matemáticos dan al dominio afectivo. Numerosos autores (matemáticos, educadores, didácticos de las matemáticas, psicólogos, filósofos) se han referido a este tema y han desarrollado investigaciones al respecto, desde sus particulares ópticas.

Es así como en las últimas décadas varios autores han escrito o han investigado acerca de estos aspectos del dominio afectivo (GOLDIN, 1988; MCLEOD; ADAMS, 1989; PONTE, 1992; CALLEJO, 1994; LLINARES, 1996; CARRILLO, 1996; CONTRERAS, 1998; FLORES, 1998; GÓMEZ-CHACÓN, 2000; GIL; RICO, 2003; GIL; BLANCO; GUERRERO, 2006; GIL; BLANCO; GUERRERO, 2005; GÓMEZ-CHACÓN; OP'T EYNDE; DE CORTE, 2006; ZAN et al. 2006; MAAß; SCHLÖGLMANN, 2009; CABALlERO; BLANCO; GUERRERO, 2011; DÍAZ; POBLETE, 2013; BLANCO; GUERRERO; CABALLERO, 2013), en relación con la importancia que adquiere su estudio en la resolución de problemas, la formación de profesores y otros aspectos de la enseñanza y aprendizaje de las Matemáticas.

En efecto, desde la década de los '80, diversos investigadores dan cuenta de la importancia que tiene el estudio de los afectos en las Matemáticas y, en particular, sobre la resolución de problemas; sin embargo, declaran que la investigación al respecto ha sido escasa aun cuando la relación entre las emociones y los procesos cognitivos, en la enseñanza y aprendizaje de las Matemáticas, se hace visible en diversas instancias: en los momentos de comprensión de la estructura o de recuperación de la información en una tarea matemática específica, o en los momentos en que se diseñan estrategias para la solución de problemas, incluyendo el recuerdo de fórmulas o procedimientos rutinarios, o en los procesos de control y autorregulación del aprendizaje unido a una metodología sobre la enseñanza de las Matemáticas que rechazan (BLANCO; CABALLERO; GUERRERO, 2009). Por lo tanto, estudiar las creencias, actitudes y emociones de los estudiantes, cuando abordan la resolución de problemas, resulta muy pertinente.

De acuerdo a Pino (2012), en la cultura escolar prevalecen una serie de categorías sociales que emergen como opiniones de estudiantes, padres y profesores, que dan cuenta de 
una serie de sentimientos a veces contradictorios, acerca de lo que es la matemática y los sentimientos que despierta en ellos. Existe una mala imagen acerca de las Matemáticas que es promovida por padres y estudiantes y, que influye en la percepción acerca de su aprendizaje.

Ha sido la propia sociedad que se ha encargado de promover la idea que la Matemática es algo muy difícil y complicado y, por tanto, sólo accesible a mentes privilegiadas o a los más inteligentes. Considerando que las Matemáticas siempre se han vinculado a la racionalidad, a los sistemas formales, a la abstracción y a la lógica, pueden parecernos extrañas las preguntas ¿Son las Matemáticas algo emocional?, ¿Existen emociones en las Matemáticas?, y si fuera necesario darle respuesta a estas preguntas planteadas, la mayoría de la gente respondería que no (GIL; BLANCO; GUERRERO, 2006). Quizá contestarían que las Matemáticas son una ciencia abstracta, rigurosa y exacta. Difícilmente podrían relacionarla con el ámbito afectivo, con la esfera de las emociones.

\section{Antecedentes teóricos}

Si son escasos los estudios sobre dimensión afectiva y aprendizaje de las Matemáticas, aún son más raros los relativos al estudio de la emoción. Las razones que parecen estar en la base de esta ausencia de trabajos son, por un lado, la gran dificultad de su diagnóstico y el no disponer de instrumentos adecuados para ello, y, por otro, la dificultad de ubicarlo en un marco teórico (GÓMEZ-CHACÓN en GIL; BLANCO; GUERRERO, 2005).

Si un estudiante asume el aprendizaje de la disciplina con la creencia de que es difícil, y se enfrenta con una enseñanza que no le permite comprender los contenidos, presentará frustración y hastío hacia la materia, se indispondrá con todo lo relacionado a ella y propiciará en él pensamientos negativos respecto a sí mismo en la materia, lo que podría convertirse en una creencia negativa de sí mismo como aprendiz (GAMBOA, 2014).

\subsection{Dimensión afectiva y aprendizaje de las Matemáticas}

Las emociones aplicadas al ámbito matemático han sido analizadas, primeramente, por Debellis y Goldin (1991, 1993), por Goldin (1988), por Mandler (1989) y por McLeod y Adams (1989). McLeod en Blanco, Caballero y Guerrero (2009), indica que los procesos cognitivos implicados en la resolución de problemas de Matemáticas son susceptibles a la influencia del dominio afectivo en tres áreas: creencias, actitudes y emociones. En este mismo sentido, encontramos las investigaciones de Gil, Blanco y Guerrero (2006) y Gómez-Chacón 
(2010).

Por sus características propias los problemas matemáticos son situaciones cuya solución no es alcanzable inmediatamente y en las que no existe un algoritmo conocido para que el estudiante aplique de inmediato; por lo cual se produce una especie de bloqueo y una interrupción a lo que sería una secuencia para llegar a la solución. Los psicólogos dicen que estas interrupciones y el despertar posterior de los resolutores conducen a la emoción, y estas emociones pueden ser positivas o negativas (MCLEOD; ADAMS, 1989).

Blanco et al. (2010) citan a McLeod (1992), que entiende las emociones como las respuestas afectivas caracterizadas por una alta intensidad y activación fisiológica que experimentan los alumnos, y que surgen en respuesta a una tarea matemática que tiene una carga de significado positiva o negativa para ellos.

Cuando a los estudiantes se les da problemas no rutinarios de Matemáticas para resolver, sus reacciones a menudo incluyen mucho de emoción. Si ellos trabajan el problema durante un período extenso de tiempo, las respuestas emocionales, frecuentemente, pueden llegar a ser muy intensas.

Muchos estudiantes empiezan a trabajar en un problema con cierto entusiasmo, tratándolo como un rompecabezas o un juego. Después de algún tiempo, aparecen reacciones más negativas. La influencia de la afectividad sobre la resolución de problemas varía en su intensidad y en su dirección (positiva o negativa). Una de las más comunes reacciones expresadas por los estudiantes es la frustración de quedarse pegado, reacción que es frecuentemente negativa e intensa.

Entre las reacciones positivas que mencionan los estudiantes se encuentra la experiencia de satisfacción, la que también es percibida intensamente (MCLEOD; ADAMS, 1989). Obviamente que la importancia de las emociones positivas en estudiantes y profesores es muy importante para el éxito en la resolución de problemas de Matemáticas. Sin embargo, los que resuelven problemas son frecuentemente inconscientes de las emociones que los están influenciando en el proceso de resolver un problema.

Schoenfeld (1992), ha escrito acerca de la gestión y los sistemas de control que los resolutores de problemas utilizan cuando abordan problemas matemáticos. En su investigación él enfatiza el rol que los sistemas de creencias juegan para determinar las decisiones ejecutivas que toman los resolutores. Además de las estrategias, Schoenfeld (1992) centra su atención en la incorporación de nuevos componentes de la resolución de problemas que puedan explicar las actuaciones de los resolutores: conocimiento base, aspectos metacognitivos, aspectos afectivos y el sistema de creencias y prácticas. 
En este último sentido, Callejo y Carrillo (2001), postulan que el estudio de la heurística fue evolucionando y complementándose con otros aspectos relacionados con el dominio afectivo, como los que menciona Schoenfeld (1992) tratando de integrar la cognición y el afecto, explorando sobre la formación y la evolución de las creencias de los alumnos y de los profesores, analizando el papel y los tipos de interacciones en el trabajo en grupo etc.

Para Céspedes (2013) las actividades de enseñanza y de aprendizaje, en todos los ámbitos, están guiadas por las emociones y manifiestan el actuar de las emociones en el hacer, por lo que como una componente no explorada en el proceso, lleva a que el estudiante se vea afectado por sus emociones, y por los efectos de las emociones propias y de los demás, en particular de la emocionalidad del profesor. En una relación de emociones, música y aprendizaje significativo, la experiencia de Albornoz (2009), examina la influencia del factor emocional en el aprendizaje y cómo este factor, generalmente ignorado en la práctica educativa, puede dar significado al proceso de aprendizaje.

Para Gamboa (2014) las emociones se corresponden con un fenómeno de tipo afectivo que un sujeto emite en respuesta a un suceso, interno o externo, que tiene para él una carga de significado. Para Maturana (2001), las emociones son disposiciones corporales dinámicas que definen los distintos dominios de acción en los que nos movemos, así cuando uno cambia de emoción, cambia de dominio de acción.

También existen estudios mediante los cuales se ha descubierto que las personas que tienen fobia a las Matemáticas son incapaces de inhibir todo lo que pueda distraer su atención, generando, con ello, una consumación de los recursos de la memoria de trabajo para toda clase de estímulo, sin relación con la tarea que se va a cumplir (HOPKO et al., 1998).

Siguiendo en esta línea, Novelo et al. (2015) investigaron algunas de las causas por las que se desarrolla lo que los autores han llamado la matefobia en los estudiantes de diferentes niveles educativos y sus efectos, así como las consecuencias que esta conlleva a lo largo de su vida académica. Si un estudiante asume el aprendizaje de la disciplina con la creencia de que es difícil, y se enfrenta con una enseñanza que no le permite comprender los contenidos, presentará frustración y hastío hacia la materia, se indispondrá con todo lo relacionado a ella y propiciará en él pensamientos negativos respecto a sí mismo en la materia, lo que podría convertirse en una creencia negativa de sí mismo como aprendiz (GAMBOA, 2014).

Para Chabot (2009) el motivo por el cual es difícil razonar con alguien que está en crisis emocional, viene del hecho de que la emocionalidad no conoce el lenguaje, por lo que no lo escucha ni lo comprende. No entiende las palabras, ya que el lenguaje es propio de lo racional. 
La definición del constructo de emoción es ardua, dada la dificultad de acotación del concepto, su multidimensionalidad y el problema de formalizar una teoría integrada que considere todas las perspectivas de estudio y dé cuenta de todos los elementos y relaciones de manera descriptiva, explicativa y predictiva.

El marco teórico que sirve de base a esta investigación, a partir de la ciencia cognitiva, se centra en el Dominio Afectivo de la Matemática según McLeod (1994), refiriéndose al mismo como un extenso rango de sentimientos y humores o estados de ánimo que son, generalmente, considerados como algo diferente de la pura cognición e incluye como componentes específicos de este dominio las actitudes, creencias y emociones. Las investigaciones de McLeod tienen como base la teoría de Mandler, quién aplicó sus propuestas a la enseñanza y al aprendizaje de la resolución de problemas en Matemáticas (MANDLER, 1984, 1989).

A continuación se presenta, en la Figura 1, los descriptores básicos; actitudes, creencias y emociones en el dominio afectivo en Matemáticas propuestos por McLeod (1994).

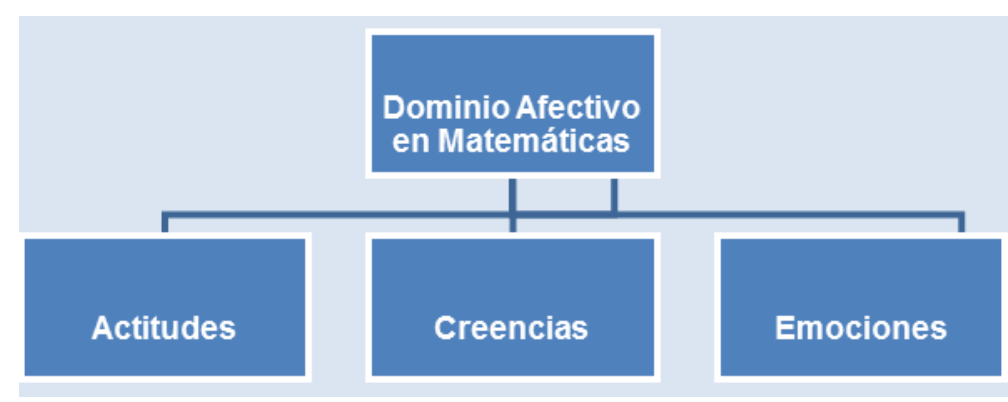

Figura 1 - Dominio Afectivo en Matemáticas y Descriptores Fuente: McLeod (1994).

Para la presente investigación, se entenderán las emociones como las respuestas afectivas caracterizadas por una alta intensidad y activación fisiológica que experimentan los alumnos, y que surgen en respuesta a una tarea matemática que tiene una carga de significado positiva o negativa para ellos (MCLEOD, 1992).

\subsection{Modelación y resolución de problemas}

Entre las competencias Matemáticas básicas se encuentran la resolución de problemas y la modelación matemática. Ambas están estrechamente unidas. Pérez (2015), tras obtener un modelo matemático de la elipse, basado en la resolución de un problema concluyó que, una vez obtenido el modelo matemático, este pasa a formar parte del conocimiento de cada estudiante y se incorporará a un campo teórico conocido como cónicas, que será aplicado 
posteriormente en la resolución de otros problemas y en la obtención de nuevos modelos.

Dentro de la Educación Matemática o Matemática Educativa existen diversas concepciones de ver a la modelación, por ejemplo, la modelación como un proceso de representación (MOCHÓN, 1997), o la modelación es vista como una forma de actividad necesaria para la reconstrucción de significados matemáticos (SUÁREZ; CORDERO, 2008). Para Biembengut y Hein (2004) la modelación es vista como método de enseñanza de las Matemáticas, en otros casos la modelación es vista como un proceso mediante el cual se desarrollan capacidades (ARAVENA; CAAMAÑO; GIMÉNEZ, 2008), o bien, la modelación es vista como una herramienta didáctica para la construcción de conceptos matemáticos (VILLA; RUIZ, 2009).

Para Molina y Arrieta (2011) la modelación, particularmente concebida, ha sido sugerida como una práctica que posibilita el establecimiento de puentes entre la escuela y su entorno, y que la constitución de dichas prácticas en los sistemas escolares, dará pauta a la vinculación. Para Zöttl, Ufer y Reiss (2011) es concebida como una competencia y una herramienta para desarrollar competencias Matemáticas.

La modelación matemática, en Chile, se aborda desde una óptica curricular integrada en los planes de estudio. Se considera que modelar es construir un modelo físico o abstracto que capture parte de las características de una realidad para poder estudiarla, modificarla y/o evaluarla; asimismo, ese modelo permite buscar soluciones, aplicarlas a otras realidades (objetos, fenómenos, situaciones etc.), estimar, comparar impactos y representar relaciones. La finalidad es que los alumnos aprenden a usar variadas formas para representar datos, y a seleccionar y aplicar los métodos matemáticos apropiados y las herramientas adecuadas para resolver problemas. De tal modo que las ecuaciones, las funciones y la geometría cobren un sentido significativo para ellos (MINEDUC, 2013).

Por otra parte, en los programas vigentes de Matemáticas en Chile, se introduce la noción de función como un cambio lineal, y sus representaciones toman mayor fuerza en la habilidad de modelar situaciones de la vida diaria y de otras asignaturas, considerando problemas abiertos y que pueden ser resueltos por medio de la función y de sus representaciones (MINEDUC, 2015). Se inicia con el empleo de formas simples de modelamiento, continuando en la enseñanza secundaria con la aplicación de modelos lineales que representan la relación entre variables hasta modelar situaciones diversas a través de funciones (DÍAZ; PÉREZ, 2016).

Investigaciones sobre la enseñanza y el aprendizaje del objeto matemático función lineal han evidenciado que existen dificultades relacionadas, principalmente, con las 
representaciones y el significado de los atributos (coeficientes), por ejemplo: Azcárate y Deulofeu (1996), Sierpinska (1992), Sastre, Rey y Boubée (2008) han manifestado que tradicionalmente en la escuela los maestros centran su interés en mostrar el aspecto algebraico del concepto dejando de lado, en muchas ocasiones, un análisis profundo y detallado sobre los elementos propios que permitan consolidar un concepto con suficiente significado para ser aprendido convenientemente. Consecuencia de esto es que los estudiantes, en muchos casos, terminan teniendo la posibilidad de repetir rutinas sobre objetos algebraicos que poco sentido tienen para ellos.

En este marco, surge la siguiente interrogante guía de la investigación: ¿es posible formular en Matemáticas una situación problema que permita dar cuenta del estado emocional del resolutor? Responder a esta interrogante implica manejar nociones teóricas provenientes del dominio afectivo y cognoscitivo e ilustrar la incorporación de la resolución de problemas en Matemática.

\section{Objetivo}

A partir de esta pregunta de investigación, formulamos como objetivo vincular aspectos cognitivos y afectivos en la resolución de una situación de aprendizaje, que permitan conocer la manifestación emocional que tienen estudiantes de tercer año de enseñanza secundaria de la Región Metropolitana de Santiago de Chile, en el proceso de aprehender el modelamiento del objeto matemático función lineal.

\section{Hipótesis}

En relación con el objetivo, se formularon las siguientes hipótesis:

$\mathrm{H}_{1}$ : Existe una relación entre la emocionalidad y el rendimiento en matemática

$\mathrm{H}_{2}$ : Las mujeres manifiestan mayor emocionalidad que los varones

$\mathrm{H}_{3}$ : El rendimiento en matemáticas de las mujeres es similar al de los hombres

$\mathrm{H}_{4}$ : El rendimiento en matemáticas de los colegios es el mismo entre ellos

$\mathrm{H}_{5}$ : El rendimiento en matemáticas de las mujeres y hombres es el mismo por cada colégio

\section{Metodología}

La presente investigación es un estudio descriptivo con metodología de trabajo 
cuantitativa y se desarrolló durante el periodo escolar 2016. La población de interés general fueron los estudiantes de educación secundaria pertenecientes a seis colegios con dependencia administrativa de financiamiento compartido de la Comuna de Maipú, en la Región Metropolitana en Santiago de Chile. Por su parte, la muestra corresponde a grupos intactos y su tamaño quedó formada por 349 estudiantes, de los cuales 182 corresponden a mujeres y 167 a hombres, de doce cursos mixtos (seis terceros A y seis terceros B) de la enseñanza secundaria modalidad científico-humanista.

\subsection{Instrumento de recogida de datos}

El instrumento evaluativo utilizado consistió en una situación de aprendizaje con doce preguntas orientada a la resolución de un problema de modelación matemática de la Ley de Hooke, posterior a haber realizado un experimento con un resorte. Esta práctica parte de datos numéricos, obtenidos de la interacción con el fenómeno, para establecer redes de modelos con el fenómeno.

La situación problema planteada fue la siguiente:

Se va a investigar la elasticidad de un resorte. Se tiene un soporte universal y un resorte colgando de él. En su extremo se le coloca una porta pesas que contiene un indicador, una flecha que apunta a una regla y se cuenta con seis pesas de 20 gramos cada una. Entonces, colocando pesas y leyendo las posiciones de la flecha del porta pesas, se elaboró una tabla como se muestra en la figura.

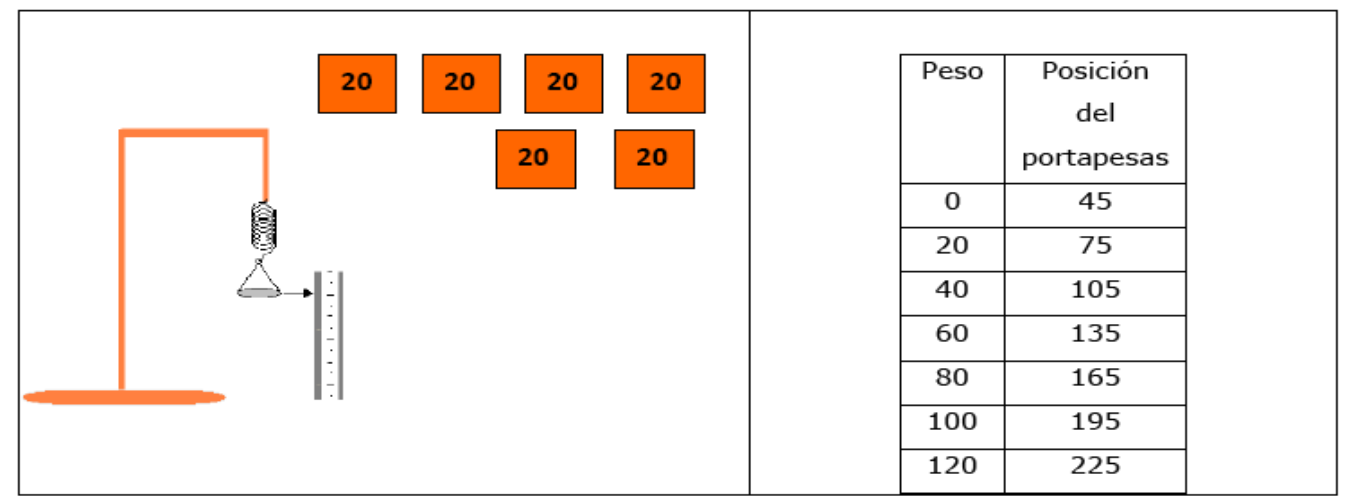

Figura 2 - Planteamiento de la situación de aprendizaje Fuente: Elaboración propia adaptada de Arrieta (2003).

La secuencia de experimentación se desarrolla desde la pregunta $\mathrm{N}^{\circ} 1$ a la pregunta $\mathrm{N}^{\circ} 11$, proponiendo cuatro actividades a los estudiantes:

(1) describir el fenómeno con sus propias palabras

(2) preguntar acerca de la posición del resorte cuando se coloca un peso que se da en la tabla 
(3) preguntar sobre el peso de la porta pesas de acuerdo a la posición del indicador

(4) representar la relación de las variables en una fórmula

\subsection{Interacción emocional}

Con el fin de conocer las emociones experimentadas por los estudiantes durante la aplicación del instrumento evaluativo, en forma individual y una vez desarrollada las once preguntas asociadas a la secuencia de experimentación, el estudiante realizaba una autovaloración de su emocionalidad respecto a la actividad de aprendizaje realizada y medida según la siguiente escala tipo Likert en la pregunta $\mathrm{N}^{\circ} 12$ : ¿Cómo te sentiste desarrollando esta actividad? [ ] Responde de 1 a 5, según la siguiente escala.

\begin{tabular}{|l|c|}
\hline \multicolumn{1}{|c|}{ Manifestación emocional } & Puntaje \\
\hline Muy bien o excelente & 1 \\
\hline Bien & 2 \\
\hline Emociones moderadas & 3 \\
\hline Desanimado o medianamente mal & 4 \\
\hline Mal o pésimo & 5 \\
\hline
\end{tabular}

Cuadro 1 - Escala de manifestación emocional Fuente: Elaboración propia.

No se colocó una opción de neutro, de manera que el estudiante opte por una opción de emocionalidad. Lo que se busca en el estudiante con la escala emocional es, que éste se plantee ser su propio observador de su estado emocional, y luego pueda declararla frente a la secuencia, para así poder analizar el fluir emocional discreto en cada estudiante, en el proceso de la modelación matemática.

\section{Análisis de la información y resultados}

Los resultados del rendimiento en la situación de aprendizaje orientada a la resolución de un problema de modelación matemática de la Ley de Hooke, posterior a haber realizado un experimento con un resorte, y los resultados de la autovaloración de su emocionalidad, obtenidos por los estudiantes, se muestran a continuación.

En primer término y concluida la investigación, se procedió a contrastar las hipótesis y atendiendo a las características de las variables rendimiento en Matemáticas, género y emocionalidad, se utilizaron pruebas de comparación de medias $\mathrm{T}$ para muestras independientes y ANOVA de un factor, con estadísticos asociados para determinar la 
dependencia de las variables por medio de la prueba Chi-Cuadrado, además se realizó una comparación múltiple con la prueba de Tukey.

Para la $\mathrm{H}_{1}$ se categorizó la variable rendimiento en matemática según puntaje obtenido en las 11 preguntas de Matemáticas de la siguiente forma (Cuadro 2):

\begin{tabular}{|l|l|}
\hline \multicolumn{1}{|c|}{ Puntajes obtenidos } & \multicolumn{1}{c|}{ Categoría } \\
\hline $0-1-2-3$ & {$[$ Entre 0 y 3] } \\
\hline $4-5-6-7$ & {$[$ Entre 4 y 7] } \\
\hline $8-9-10-11$ & {$[$ Entre 8 y 11] } \\
\hline
\end{tabular}

Cuadro 2 - Categorías variable rendimiento en matemática

Fuente: Elaboración propia.

La variable emocionalidad fue categorizada en tres niveles: positiva, moderada y negativa (Cuadro 3).

\begin{tabular}{|c|c|}
\hline Respuestas en escala & Categoría \\
\hline $1-2$ & Positiva \\
\hline 3 & Moderada \\
\hline $4-5$ & Negativa \\
\hline
\end{tabular}

Cuadro 3 - Categorías variable emocionalidad Fuente: Elaboración propia.

La tabla de contingencia de 3x3 (Tabla 1) permite observar la relación de las variables medidas en escalas de negativo a positivo.

Tabla 1 - Nivel de logro y escala de emociones

\begin{tabular}{|c|c|c|c|c|c|c|}
\hline & & & & vel de Logro & & Total \\
\hline & & & $\begin{array}{c}\text { [Entre } 9 \text { y } 12 \\
\text { puntos] }\end{array}$ & $\begin{array}{c}\text { [Entre } 5 \text { y } 8 \\
\text { puntos] }\end{array}$ & $\begin{array}{c}\text { [Entre } 1 \text { y } 4 \\
\text { puntos] }\end{array}$ & \\
\hline & Positiva & $\begin{array}{l}\text { Recuento } \\
\% \text { del total }\end{array}$ & $\begin{array}{r}93 \\
\mathbf{2 6 , 6 \%} \\
\end{array}$ & $\begin{array}{r}24 \\
6,9 \% \\
\end{array}$ & $\begin{array}{r}14 \\
\mathbf{4 , 0 \%} \\
\end{array}$ & $\begin{array}{r}131 \\
\mathbf{3 7 , 5 \%} \\
\end{array}$ \\
\hline $\begin{array}{l}\text { Escala } \\
\text { Emocional }\end{array}$ & Moderada & $\begin{array}{l}\text { Recuento } \\
\% \text { del total }\end{array}$ & $\begin{array}{r}50 \\
14,3 \% \\
\end{array}$ & $\begin{array}{r}26 \\
7,4 \% \\
\end{array}$ & $\begin{array}{r}17 \\
\mathbf{4 , 9 \%} \\
\end{array}$ & $\begin{array}{r}93 \\
\mathbf{2 6 , 6 \%} \\
\end{array}$ \\
\hline & Negativa & $\begin{array}{l}\text { Recuento } \\
\% \text { del total }\end{array}$ & $\begin{array}{r}58 \\
16,6 \% \\
\end{array}$ & $\begin{array}{r}33 \\
\mathbf{9 , 5 \%} \\
\end{array}$ & $\begin{array}{r}34 \\
\mathbf{9 , 7 \%} \\
\end{array}$ & $\begin{array}{r}125 \\
\mathbf{3 5 , 8 \%} \\
\end{array}$ \\
\hline Total & & $\begin{array}{l}\text { Recuento } \\
\% \text { del total }\end{array}$ & $\begin{array}{r}201 \\
57,6 \% \\
\end{array}$ & $\begin{array}{r}83 \\
\mathbf{2 3 , 8 \%} \\
\end{array}$ & $\begin{array}{r}65 \\
18,6 \% \\
\end{array}$ & $\begin{array}{r}349 \\
\mathbf{1 0 0 , 0 \%} \\
\end{array}$ \\
\hline
\end{tabular}

Fuente: Elaboración propia.

De acuerdo a la Tabla 1, a medida que las emociones son positivas, los alumnos obtienen mayor rendimiento, en este sentido se tiene que el $26,6 \%$ de los estudiantes tuvieron un puntaje en matemática entre 8 y 11 puntos y categorizaron su emoción en un estado positivo (se sintieron bien o muy bien desarrollando la actividad). Se observa que mientras menor puntaje obtuvieron los alumnos, su emoción se manifestaba de forma negativa. Al realizar la prueba Chi-Cuadrado para determinar independencias de variables (al nivel de significancia del 0,001) se acepta la hipótesis $\mathrm{H}_{1}$ entre el nivel de logro y el nivel de emocionalidad. Por tanto se tiene que los niveles obtenidos de logro en Matemática dependen 
del nivel de emocionalidad obtenidos.

Las medidas simétricas de concordancia entre escala emocional y nivel de logro muestran que existe una asociación ligera y positiva, a mayor puntaje de logro mayor estado emocional y viceversa. El coeficiente de correlación de Spearman es de 0,226 con un error típico asintótico de 0,051 ; por tanto, es significativa a cualquier nivel de significancia. Por otro lado, las categorías moderada y negativa en estado emocional presentan porcentajes similares, por lo que los estadísticos no muestran una relación muy pronunciada. Aun así se evidencia que el mayor porcentaje de respuesta de los estudiantes queda en la intersección de los mejores puntajes y más altos niveles de emoción.

\subsection{Análisis de la Manifestación Emocional}

A continuación se presentan los resultados obtenidos del análisis de la totalidad de la muestra que corresponde a 349 sujetos de estudio, en función de la externalización del estado emocional en la resolución del problema de modelación matemática. La Figura 3 detalla la frecuencia de la totalidad de las respuestas distribuidas en función de la escala de 1 a 5 puntos utilizada.

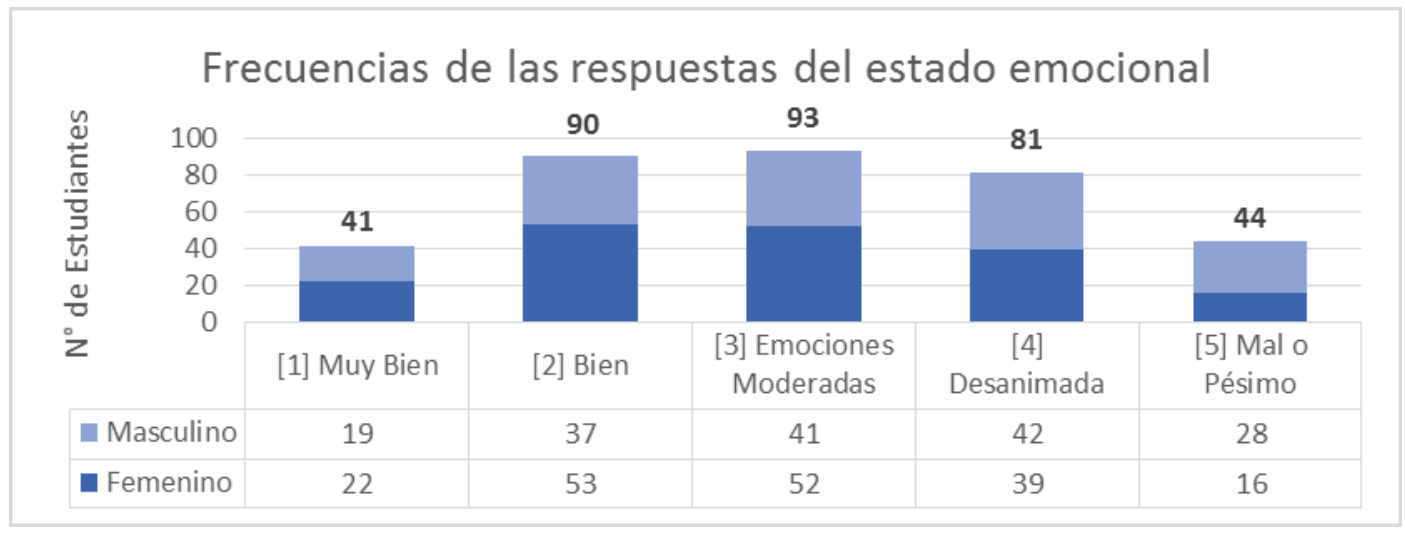

Figura 3 - Frecuencia de respuesta de estado emocional Fuente: Elaboración propia.

En la Figura 3 se observa cierta simetría entre la manifestación de emociones positivas y negativas, dado que 131 estudiantes al término de la actividad de aprendizaje, declaran sentirse muy bien y bien, en tanto que 125 alumnos (as) dicen sentirse desanimados (as) y mal o pésimo al realizar la actividad. Sin embargo, se observa una mayoría de frecuencias de estado emocional en las emociones moderadas con 83 estudiantes. En términos porcentuales, esta comparación se muestra en la Figura 4. 


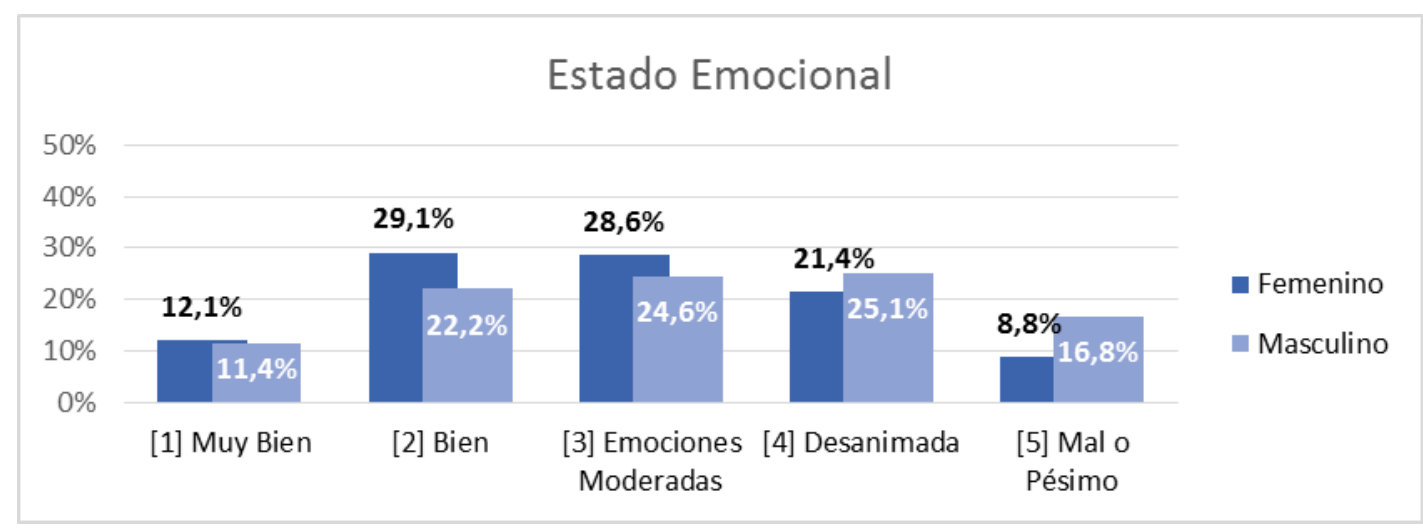

Figura 4 - Respuestas por estado emocional Fuente: Elaboración propia.

De acuerdo a la Figura 4, los porcentajes de respuesta por género asociados a emociones positivas son $41,2 \%$ (muy bien y bien, mujeres) y 33,6\% (muy bien y bien, hombres), mayores que los porcentajes de respuesta asociados a emociones negativas, que son $29,7 \%$ (desanimada, mal o pésimo, mujeres) y 41,9\% (desanimado, mal o pésimo, hombres). Con emociones moderadas el $28,6 \%$ (mujeres) y 24,6\% (hombres). Por lo tanto, se puede observar que en el género femenino existen emocionalidades levemente más altas que entre los hombres, sin embargo es necesario determinar si estas diferencias son significativas. En base a una tabla de contingencia que relacionó las variables género y emocionalidad, se elaboró la Figura 5.

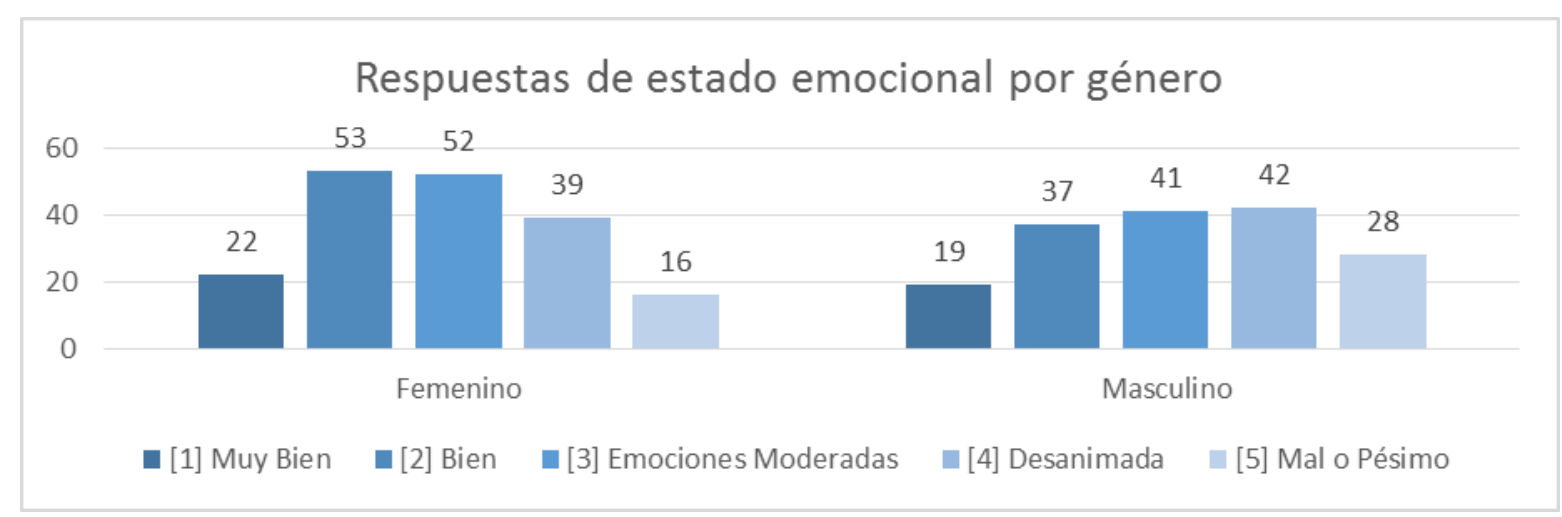

Figura 5 - Respuestas de estado emocional por género Fuente: Elaboración propia.

Al aplicar una prueba Chi-Cuadrado para determinar la relación entre la variable género y nivel emocional, como conclusión con un nivel de confianza del 95\% se rechaza la hipótesis $\mathrm{H}_{2}$ en la que existe relación entre la variable género y la manifestación emocional $(\operatorname{sig}=0,13)$.

\subsection{Análisis del rendimiento en matemáticas}


A continuación se presentan los resultados en el rendimiento en la situación de aprendizaje orientada a la resolución de un problema de modelación matemática. En la Tabla 2 se detallan los puntajes obtenidos por los estudiantes distribuidos por género.

Tabla 2 - Puntajes de logro por género

\begin{tabular}{|c|c|c|}
\hline Puntaje & Mujeres & Hombres \\
\hline 0 & 3 & 9 \\
\hline 1 & 8 & 4 \\
\hline 2 & 8 & 8 \\
\hline 3 & 12 & 13 \\
\hline 4 & 14 & 13 \\
\hline 5 & 8 & 8 \\
\hline 6 & 11 & 7 \\
\hline 7 & 11 & 11 \\
\hline 8 & 24 & 14 \\
\hline 9 & 24 & 23 \\
\hline 10 & 35 & 36 \\
\hline 11 & 24 & 21 \\
\hline Total & 182 & 167 \\
\hline
\end{tabular}

A continuación, la Figura 6 muestra a simple vista que las distribuciones en el rendimiento de matemática entre hombres y mujeres son muy similar, sin embargo se aplicó una prueba $\mathrm{T}$ de comparación de medias para determinar si existen diferencias significativas entre ellos.

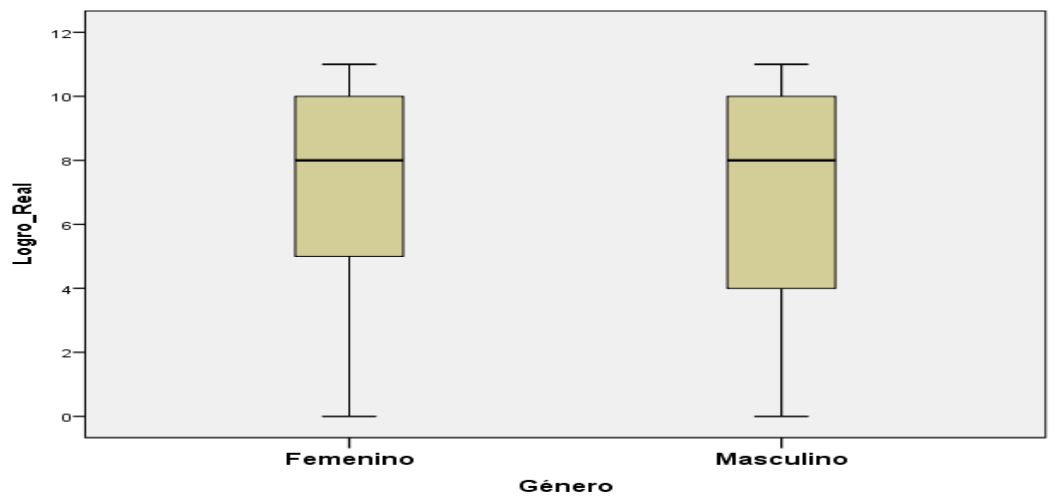

Figura 6 - Puntajes de logro por género Fuente: Elaboración propia.

Al realizar una prueba de $\mathrm{T}$ de comparación de medias se tiene que con una significancia de contraste del $5 \%(\mathrm{sig}=0,584)$, se puede concluir que no se rechaza la hipótesis $\mathrm{H}_{3}$ ya que no existen diferencias significativas entre hombres y mujeres en el rendimiento en Matemáticas (Tabla 3).

Tabla 3 - Estadísticos de grupo

\begin{tabular}{|l|c|c|c|c|}
\hline \multicolumn{1}{|c|}{ Género } & $\mathbf{N}$ & Media & Desviación Típica & $\begin{array}{c}\text { Error tip. de la } \\
\text { media }\end{array}$ \\
\hline Femenino & 182 & 7,26 & 3,156 & 0,234 \\
\hline Masculino & 167 & 7,07 & 3,396 & 0,263 \\
\hline
\end{tabular}

Fuente: Elaboración propia. 


\subsection{Análisis del rendimiento por colegio}

A continuación, la Figura 7 muestra que a simple vista hay diferencias de logros obtenidos entre algunos colegios, sin embargo es necesario determinar si estas diferencias son significativas.

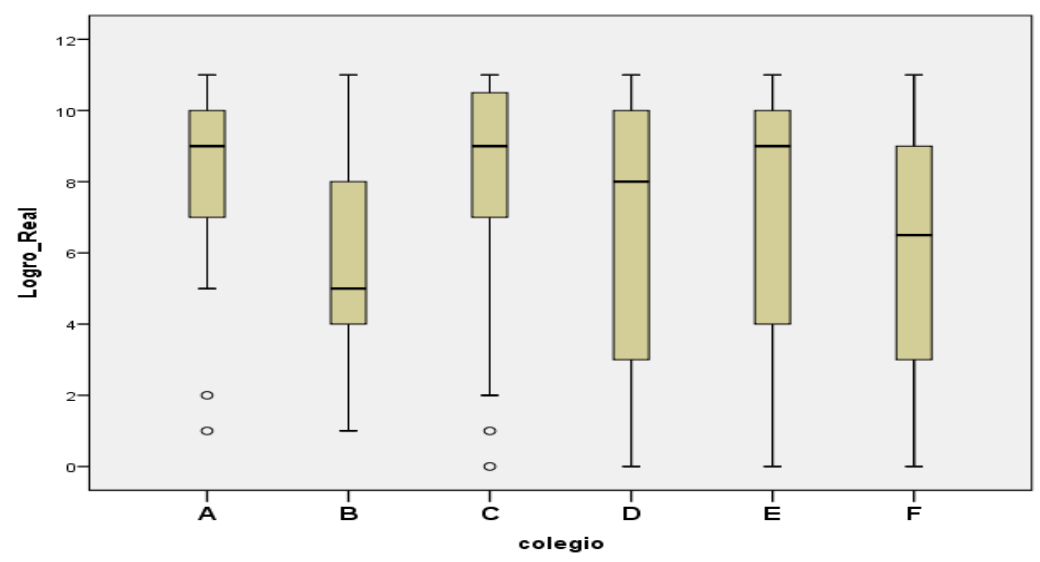

Figura 7 - Rendimiento en matemática por colegio Fuente: Elaboración propia.

Para ello se aplicó un ANOVA de un factor, seguido de una prueba de Tukey para determinar diferencias por cada par de colegio. Los resultados no apoyan la hipótesis $\mathrm{H}_{4}$ respecto a que los colegios tienen distinto nivel de logro en Matemáticas. De acuerdo a la estadística de prueba de Levene para determinar varianzas homocedásticas entre los colegios, este concluye que existen varianzas iguales entre ellos ( $\operatorname{sig}>0,005)$.

En cuanto a la comprobación de la hipótesis $\mathrm{H}_{5}$, como puede verse a continuación, en la Figura 8, al analizar el Box Plot, se observa que los colegios mantienen una concentración similar del puntaje obtenido en Matemáticas entre hombres y mujeres, a excepción del colegio A y B y las mujeres del colegio $\mathrm{C}$.

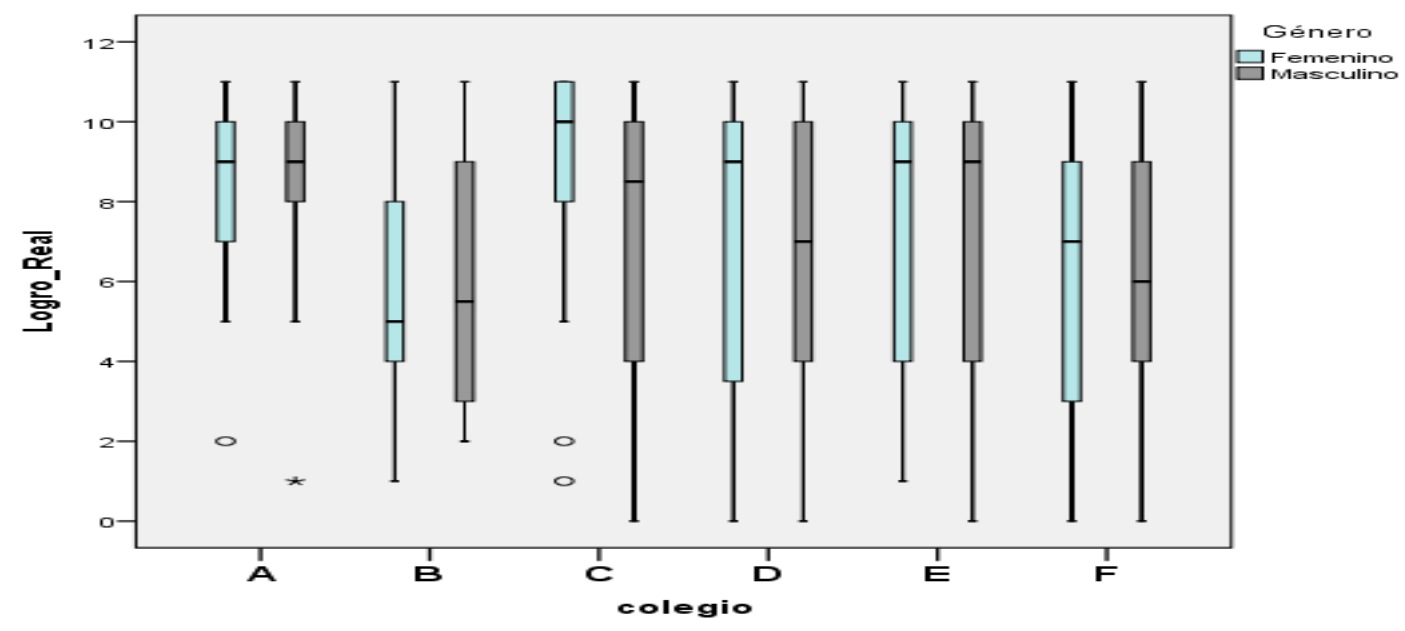

Figura 8 - Rendimiento en matemática por colegio Fuente: Elaboración propia. 
Al realizar una prueba ANOVA de un factor, no se acepta la hipótesis H5 basados en la igualdad de medias entre colegios y género.

\section{Conclusiones}

Es una realidad que las emociones intervienen en el aprendizaje de manera significativa ya sea facilitándolo u obstaculizándolo, desempeñando un papel en la comunicación de intenciones de los estudiantes hacia los demás; pero en el ámbito de la investigación en Educación Matemática, los aspectos afectivos todavía no encuentran un eco amplio, por lo que se han realizado pocas investigaciones que se centran en esta dimensión. En gran parte, esto tal vez se deba al popular mito de que las Matemáticas son algo puramente intelectual, donde el comportamiento relativo a las emociones no desempeña un papel esencial.

Ante el frecuente fracaso escolar de los estudiantes, que muchas veces no corresponde a su desenvolvimiento cognitivo, a través del desarrollo de esta investigación, se buscó integrar la perspectiva afectiva y cognitiva a las situaciones de enseñanza-aprendizaje, aportando en el ámbito del estudio de las emociones en el aprendizaje de las Matemáticas y tocando un tema de gran actualidad, ya que, como es sabido, la necesidad de descifrar los aspectos emocionales del conocimiento de la Matemática crece día con día, pues se consideran como la fuente de muchos fracasos en la vida intelectual y, en particular, en la educación.

La formulación de preguntas es una herramienta potente para propiciar el aprendizaje científico, en especial cuando la interrogante se orienta hacia la indagación o investigación respecto a la posibilidad de formular en matemática una situación problema que permita dar cuenta del estado emocional del estudiante. Para responder específicamente a esta pregunta, se utilizó un instrumento evaluativo adaptado para esta investigación, que consistió en una situación de aprendizaje orientado a la resolución de un problema de modelación matemática de la Ley de Hooke, posterior a haber realizado un experimento con un resorte, con el objetivo de conocer la capacidad para resolverlo y conocer las emociones que se generaban durante la actividad.

Se utilizó la modelación matemática entendiéndola como un intento de describir una parte del mundo real en términos matemáticos. Modelos matemáticos han sido construidos en todas las ciencias tanto físicas, como biológicas y sociales. Los elementos que lo componen 
son tomados del cálculo, el álgebra, la geometría y otros campos afines. La creación de modelos matemáticos, es un área de la ciencia que se encarga de expresar fenómenos de la vida real en forma matemática, permitiendo, así, usar las herramientas que ofrecen las Matemáticas para obtener solución a un determinado problema (YOIS, 2015).

Los datos obtenidos en esta investigación y sus correspondientes análisis estadísticos apoyaron las hipótesis $\mathrm{H}_{1}$ y $\mathrm{H}_{3}$, y refutaron las $\mathrm{H}_{2}, \mathrm{H}_{4}$ y $\mathrm{H}_{5}$, por tanto se puede decir que existe una relación entre la emocionalidad y el rendimiento en matemática de los estudiantes de la enseñanza secundaria al trabajar en una situación de aprendizaje, orientada a la resolución de un problema de modelación matemática de la Ley de Hooke, posterior a haber realizado un experimento con un resorte, y autovalorar su emocionalidad respecto al desarrollo de la actividad. Además, el rendimiento en matemáticas de los seis colegios participantes de la investigación es similar entre ellos y hay diferencias significativas entre el rendimiento en matemáticas de las mujeres y hombres por cada colegio.

A partir de los significados expuestos en el marco teórico, se puede deducir, que la emocionalidad individual es el conjunto de emociones que concurren en un sujeto en particular en un instante dado, bajo la percepción de ciertos estímulos a través de los órganos de los sentidos, o de recuerdos que puedan actuar como activadores emocionales.

De acuerdo a los resultados, el nexo entre aprendizaje y emociones se vuelve aquí evidente. Cada vez que la atención está monopolizada por una carga emocional negativa, el aprendizaje y el desempeño se afectan (CHABOT, 2009). Y a la inversa, se puede complementar que, cada vez que el aprendizaje se ve afectado por una emoción positiva, éste se potencia de manera retroalimentada, es decir, se motiva al alumno y al estar motivado, entonces, aprende más, y al aprender más, aumenta su motivación. Es un círculo virtuoso.

Con este trabajo se ha logrado un vínculo de aspectos cognitivos y afectivos en la resolución de una situación de aprendizaje, que ha permitido conocer la manifestación emocional que tienen estudiantes de tercero año de secundaria pertenecientes a colegios científico- humanistas y dependencia particular con financiamiento compartido de la Comuna de Maipú en la Región Metropolitana, en el proceso de aprehender el objeto matemático en la modelación lineal, confirmándose, de esta manera, el cumplimiento del objetivo general de este estudio.

\section{Referencias}

ALBORNOZ, Y. Emoción, música y aprendizaje significativo. Educere, Mérida, v. 13, n. 44, p. 67 73, 2009. 
ARAVENA, M.; CAAMAÑO, C.; GIMÉNEZ, J. Modelos matemáticos a través de proyectos. Relime, México DF, v. 11, n. 1, p. 49-92, 2008.

ARRIETA, J. Las prácticas de modelación como proceso de matematización en el aula. Tesis doctoral no publicada del Departamento de Matemática Educativa, Cinvestav-IPN, México DF, 2003.

AZCÁRATE, C.; DEULOFEU, J. Funciones y Gráficas. Madrid: Síntesis, 1996.

BIEMBENGUT, M.; HEIN, N. Modelación matemática y los desafíos para enseñar matemática. Educación Matemática, México DF, v. 16, n. 2, p. 105-125, 2004.

BLANCO, L. J.; CABALLERO, A.; GUERRERO, E. El dominio afectivo en la construcción del conocimiento didáctico del contenido sobre resolución de problemas de matemáticas. Enseñanza de las Ciencias, Barcelona, n. extra, p. 362-365, 2009.

BLANCO, L. et al. El Domino afectivo en la enseñanza/aprendizaje de las matemáticas. Una revisión de investigaciones locales. Campo Abierto, Badajoz, v. 29, n. 1, p. 15-33, 2010.

BLANCO, L. J.; GUERRERO, E.; CABALLERO, A. Cognition and affect in mathematics problem solving with prospective teachers. The Mathematics Enthusiast, Missoula, v. 10, n. 1 y 2, p. 335364, 2013.

CABALLERO, A.; BLANCO L. J.; GUERRERO, E. Problem solving and emotional education in initial primary teacher education. Eurasia Journal of Mathematics, Science y Technology Education, London, v. 7, n. 4, p. 281-292, 2011.

CALLEJO, M. L. Un club matemático para la diversidad. Madrid: Narcea, 1994.

CALLEJO, M. L.; CARRILLO, J. Elementos de resolución de problemas, cinco años después. En: LACASTA, E; PASCUAL, J. R. (Ed.). Investigación en Educación Matemática. Pamplona, España: SEIEM, 2001. p. 83-105.

CARRILLO, J. Modos de resolver problemas y concepciones sobre la matemática y su enseñanza de profesores de matemáticas de alumnos de más de 14 años. Algunas aportaciones a la metodología de la investigación y estudio de posibles relaciones. Epsilon: Revista de la Sociedad Andaluza Thales, Cádiz, n. 36, p. 451-454, 1996.

CÉSPEDES, A. Las Emociones van a la escuela, educar para la vida. Vergara: Santiago de Chile, 2013.

CHABOT, D. Pedagogía emocional sentir para aprender, integración de la inteligencia emocional en el aprendizaje. Alfa Omega: México, 2009.

CONTRERAS, L. C. Resolución de problemas: un análisis exploratorio de las concepciones de los profesores acerca de su papel en el aula. 1998. 529 f. Tesis (Doctorado en Psicopedagogía) Universidad de Huelva, España, 1998.

DEBELLIS. V.; GOLDIN, G. Interactions between cognition and affect in eight high school students' individual problem solving. In: ANNUAL METING OF THE NOTH AMERICAN, 13., 1991.

Virginia. Proceedings ... Blacksburg: Virginia Polytechnic Institute and State University. 1991. p. 2935 . 
DEBELLIS, V.; GOLDIN, G. Analysis of interactions between affect and cognition in elementary school children during problem solving. In: ANNUAL METING OF THE NOTH AMERICAN, 15. 1991, California. Proceedings... California: Pacific Grove, CA, 1993. p. 56-62.

DÍAZ, V.; PEREZ, I. Uso de gráficas en una situación de modelación del movimiento en matemática en la enseñanza secundaria. Paradigma, Maracay, v. 27, n. 1, p. 161-180, 2016.

DÍAZ, V.; POBLETE, A. Resolución de problemas en matemática y su integración con la enseñanza de valores éticos: el caso de Chile. Bolema, Rio Claro (SP), v. 27, n. 45, p. 117-141, 2013.

FLORES, P. Creencias y concepciones de los futuros profesores de matemáticas de enseñanza secundaria sobre las matemáticas, su enseñanza y aprendizaje. Investigación durante las prácticas de enseñanza. Comares: Granada, 1998.

GAMBOA, R. Relación entre la dimensión afectiva y el aprendizaje de las matemáticas. Revista Electrónica Educare, Heredia, v.18, n. 2, 2014.

GIL, N.; BLANCO, L.; GUERRERO, E. The affective domain in Mathematics learning. International Electronic Journal of Mathematics Education, Ankara, v. 1, n. 1, p. 16-32, 2005.

GIL, N.; BLANCO, L. J.; GUERRERO, E. El papel de la afectividad en la resolución de problemas matemáticos. Revista de Educación, Madrid, n. 340, p. 551-569, 2006.

GIL F.; RICO, L. Concepciones y creencias del profesorado de secundaria sobre enseñanza y aprendizaje de las matemáticas. Enseñanza de las Ciencias, Barcelona, v. 21, n. 1, p. 27-47, 2003.

GOLDIN, G. A. Affective representation and mathematical problem solving. In: MEETING ON THE PSYCHOLOGY OF MATHEMATICS EDUCATION, NORTH AMERICAN CHAPTER OF INTERNATIONAL GROUP, 10., 1988, Illinois. Proceedings... North Illinois University: DeKalb, IL. 1988. p. 1-7.

GOMEZ-CHACÓN, I. Matemática emocional: los afectos en el aprendizaje matemático. Madrid: Narcea, 2000.

GÓMEZ-CHACÓN, I. Actitudes de los estudiantes en el aprendizaje de la matemática con tecnología. Enseñanza de las Ciencias, Barcelona, v. 28. n. 2, p. 227-244, 2010.

GÓMEZ-CHACÓN, I. M.; OP ’T EYNDE, P.; DE CORTE, E. Creencias de los estudiantes de matemáticas, la influencia del contexto de clase. Enseñanza de las Ciencias, Barcelona, v. 24, n. 3, p. 309-324, 2006.

HOPKO, D. R. et al. Mathematics anxiety and working memory: support for the existence of a deficient inhibition mechanism. Journal of Anxiety Disorder, Regina, Saskatchewan, v. 12, n. 4, p. 343-355, 1998

LLINARES, S. Conocimiento profesional del profesor de matemáticas: conocimiento creencias y contexto en relación a la noción de función. En: PONTE, J. P. et al. (Ed.). Desenvolvimento Professional dos Professores de matemática. Que Formaçao? Lisboa: Sociedade Portuguesa de Ciencias da Educaçao, Secçao de Educaçao Matemática, 1996. p. 47-82.

MAAß, J.; SCHLÖGLMANN, W. Beliefs and Attitudes in Mathematics Education. New Research Results. Sense Publishers: Rotterdam, Taipei, 2009.

MANDLER, G. Mind and body: Psychology of emotion and stress. New York: WW Norton, 1984. 
MANDLER, G. Affect and learning: Causes and consequences of emotional interactions. En: Affect and mathematical problem solving. Springer: New York, 1989. p. 3-19.

MATURANA, H. Emociones y lenguaje en educación y política. Dolmen Ensayo: Chile, 2001. MCLEOD, D. Research on affect in the mathematics education. (Ed.). Handbook of Research on Mathematics Teaching and Learning. MacMillan: NCTM New York, 1992. p. 575-596.

MCLEOD, D. Research on affect and mathematics learning. Journal for Research in Mathematics Education, Baltimore, v. 25, n. 6, p. 637-647, 1994.

MCLEOD, D.; ADAMS, V. M. Affect and mathematical problem solving: A new perspective. New York: Springer-Verlang, 1989.

MINEDUC. Ministerio de Educación. Fundamentos Bases Curriculares 2013. Matemática. Santiago: Ministerio de Educación, 2013.

MINEDUC. Ministerio de Educación. Bases Curriculares 2015. Santiago: Ministerio de Educación, 2015.

MOCHÓN, S. Modelos matemáticos para todos los niveles. En: FARFÁN, R. M.; LEZAMA, J.; ARELLANO, A.; OAXACA, E. (Ed.). Acta Latinoamericana de Matemática Educativa 11, México: Grupo Editorial Iberoamérica, 1997. p. 42-45.

MOLINA, J.; ARRIETA, J. La Constitución de las prácticas de modelación lineal. En: Aspectos socioepistemológicos en el análisis y el rediseño del discurso matemático escolar. Acta Latinoamericana de Matemática Educativa, 2011.p. 793-803.

NOVELO, C. et al. Temor a las matemáticas: causa y efecto. Revista Iberoamericana de Producción Académica y Gestión Educativa, México DF, v. 2, n. 3, 2015. Disponible en: < http://www.pag.org.mx/index.php/PAG/article/view/268 >. Acceso en: 23 nov. 2016.

PÉREZ, R. Resolución de problemas y modelización matemática para la clase. UNO Revista de Didáctica de las Matemáticas, La Laguna, 69, p. 7-21, 2015. Disponible en: <http://uno.grao.com /revistas/uno/69-modelizacion/resolucion-de-problemas-y-modelizacion-matematica-para-la-clase> . Acceso en: 18 nov. 2016.

PINO, J. Concepciones y prácticas de los estudiantes de pedagogía media en matemáticas con respecto a la resolución de problemas y, diseño e implementación de un curso para aprender a enseñar problemas. 2012. 378 f. Tesis (Doctorado en Didáctica de las Ciencias Experimentales y de las Matemáticas) - Universidad de Extremadura, España, 2012.

PONTE, J. P. Concepcóes dos professores de matemática e processos de formacáo. En: PONTE, J. P (Ed.). Educacáo Matemática: Temas de Investigacáo, Lisboa: Instituto de Inovacáo Educacional, 1992. p. 185-239.

SASTRE, Z, P.; REY, G.; BOUBÉE, C. El concepto de función a través de la historia. Unión, Revista Iberoamericana de Educación Matemática, La Rioja, España, n. 16, p. 141-155, 2008.

SCHOENFELD, A. H. Learning to think mathematically: Problem solving, metacognition, and sensemaking in mathematics. In: GROUWS, D. (Ed.). Handbook for Research on Mathematics Teaching and Learning. New York: MacMillan, 1992. p. 334-370.

SIERPINSKA, A. On understanding the notion of function. In: HAREL G.; DUBINSKY, E. (Ed.). The Concept of Function, Aspects of Epistemology and Pedagogy. USA: Mathematical Association of America, 1992. p. 25-58. 
SUÁREZ, L.; CORDERO, F. Elementos teóricos para estudiar el uso de las gráficas en la modelación del cambio y de la variación en un ambiente tecnológico. Revista Electrónica de Investigación en Educación en Ciencias, Buenos Aires, v. 3, n. 1, p. 51-58, 2008.

VILLA, J.; RUIZ, H. Modelación en Educación Matemática. Una mirada desde los lineamientos y estándares curriculares colombianos. Revista Virtual Universidad Católica del Norte, Medellín, 27, $1-21,2009$.

YOIS, P. Modelamiento matemático aplicado al modelamiento especial. Ediciones Universidad Distrital Francisco José de Caldas: Bogotá, Colombia, 2015.

ZAN, R. et al. Affect in mathematics education: an introduction. Educational Studies in Mathematics, Dordrecht, v. 63, p. 13-121, 2006.

ZÖTTL, L.; UFER, S.; REISS, K. Assessing modelling competencies using a multidimensional IRTapproach. En: KAISER, G; BLUM, B. R.; STILLMAN, G. (Ed.). Trends in Teaching and Learning of Mathematical Modelling: ICTMA 14. New York: Springer, 2011. p. 427-437.

Submetido em 10 de Maio de 2017. Aprovado em 20 de Abril de 2018. 